\title{
José Marcos López Mojica: un inesperado adiós y un recuerdo permanente
}

\author{
Lilia P. Ake ${ }^{1}$ \\ María S. García González ${ }^{2}$ \\ Ana María Ojeda Salazar ${ }^{3}$
}

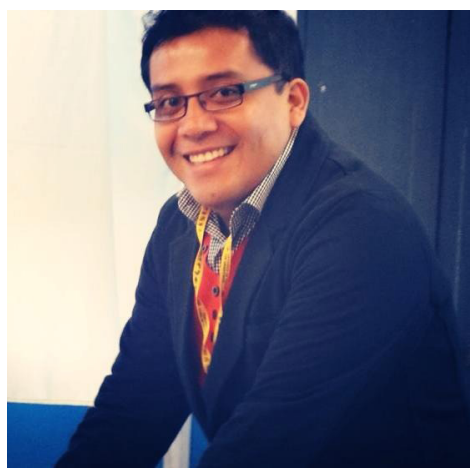

José Marcos nació el día 6 de octubre de 1984, en la ciudad de Chilpancingo de los Bravo en el estado de Guerrero. Como carrera profesional eligió la enseñanza de las matemáticas, y fue así que se matriculó en la Facultad de Matemáticas de la Universidad Autónoma de Guerrero de la ciudad que le vio nacer, y optó por especializarse en Matemática Educativa. Sus profesores en estos años, lo recuerdan como un estudiante responsable, inteligente y curioso por aprender más de los temas que se le enseñaban. José Marcos comentaba que, entre ellos, Patricia Colín lo impulsó a participar en los que fueron sus primeros congresos de Matemática Educativa, en los que encontró un espacio propicio para la discusión y reflexión de la disciplina que había elegido como profesión.

\footnotetext{
1 Universidad Autónoma de Querétaro, México. lake86@gmail.com, orcid.org/0000-0003-4303-4895.

2 Universidad Autónoma de Guerrero, México. mgargonza@gmail.com, orcid.org/0000-0001-7088-1075.

3 Cinvestav-IPN, México. amojeda@cinvestav.mx, orcid.org/0000-0001-7918-7557.
} 
La carrera de José Marcos como investigador ha sido muy corta y, sin embargo, muy talentosa y prometedora. Motivado por consolidar su formación ingresó a la maestría en Ciencias en la especialidad de Matemática Educativa del Cinvestav-IPN y al concluirla continuó con el doctorado. Su formación en la investigación fue acelerada y constante; a cada paso dio muestras de entusiasmo, perseverancia y dedicación. Al inicio de su segundo año en la maestría, en el año 2008, encauzó su interés en la problemática de la educación en estocásticos, tema que en general ha merecido poca atención, pero menos aún para ser investigado en la educación especial y todavía menos en un Centro de Atención Múltiple (CAM), sitio en el que convergen distintas discapacidades. Sus resultados obtenidos al cabo de un año de trabajo se reunieron en la tesis Estocásticos en el Segundo Año de Educación Especial, que mostró la desatención a esta modalidad de la educación, que va desde la formación del docente, al tratamiento educativo de los niños con discapacidad y a la actualización de los docentes en servicio. Sobre esta base, José Marcos, inició su investigación de doctorado, que culminó en diciembre de 2013 con la tesis Pensamiento probabilístico y esquemas compensatorios en Educación Especial. Como la anterior, la desarrolló in situ, en la complejidad de las aulas pero ahora de preescolar, de primaria y de secundaria. Su investigación estableció un marco de referencia para la enseñanza de los temas de estocásticos en la Educación Especial básica y la conveniencia de incluirlos en ella para promover el uso de esquemas compensatorios, según la discapacidad de que se tratase. Pionera en México, esta tesis doctoral originó una línea de investigación no sólo pertinente, sino apremiante por la problemática que identificó. En su corta estancia en Cinvestav como estudiante, su participación fue muy activa en los seminarios y en los coloquios, en distintos congresos internacionales y regionales en los que iba presentando sus resultados.

Ya como investigador, al integrarse al campo laboral como profesor universitario, su participación en una diversidad de actividades académicas se intensificó con paso firme, responsable y cada vez más consolidado. Fue en 2014 que se incorporó a la Universidad de Colima a través de su participación en una convocatoria de plazas a concurso abierto. En continuidad con su trabajo doctoral sobre probabilidad y discapacidad, sus investigaciones posteriores configuraron una sinergia entre la Matemática Educativa y la Educación Especial. En ese entonces, inició diversos estudios con tesistas y colegas interesados en el tema; algunos de estos trabajos se concretaron en su primer libro colaborativo: Matemática Educativa y Educación Especial. Una aproximación desde la 
formación docente y procesos de enseñanza. Se distinguía por tener un trabajo académico activo, congruente y con iniciativas: tenía presencia en congresos, realizaba estancias y entrelazaba colaboraciones con colegas de diferentes instituciones. Su fuerte compromiso institucional impulsó la obtención del Perfil Deseable y a conformar un Cuerpo Académico. Había iniciado en la investigación y como profesor universitario con paso firme y responsable.

Posteriormente, continuó su vida académica en la Universidad Autónoma de Guerrero donde se incorporó en el 2018. Con rigor teórico y metodológico abordaba una problemática emergente ante la educación inclusiva y la incorporación de las personas con discapacidad en las aulas regulares. Sus investigaciones entretejían sus intereses e inquietudes sobre los alcances y limitaciones del desarrollo del pensamiento matemático en personas con diferentes tipos de discapacidad. Sus reflexiones sobre este tema lo condujeron a trabajar, en lo que denominaría, Matemática Educativa Inclusiva, sus últimos trabajos se enfocaron en este constructo. Una segunda obra colegiada, aún sin publicar sobre esta línea de investigación es: Educación Especial en Matemática Educativa. Fundamentos teórico-metodológicos para la investigación. En este y todos los trabajos de José Marcos se sumaban esfuerzos y se teñían del mismo propósito: aproximar el estudio de las matemáticas a las personas con discapacidad orientando a la docencia desde la investigación.

Las huellas de su trayectoria le permitieron la distinción del Nivel 1 en el Sistema Nacional de Investigadores mexicanos en 2017. Para José Marcos esta distinción representó un aliciente y reconocimiento a cada uno de los trabajos realizados que ratificaba su compromiso de continuar con la generación y aplicación del conocimiento. No era la primera vez que recibía una distinción por su trabajo. En 2009 la Sociedad Matemática Mexicana reconoció como mejor tesis de maestría en Matemática Educativa su trabajo Estocásticos en el Segundo Grado de Educación Especial. En 2014 el Cinvestav otorgó el "Premio Arturo Rosenblueth" a su tesis de doctorado Pensamiento probabilístico y esquemas compensatorios en Educación Especial, misma que en 2015 fue acredora de una mención honorífica del "Premio Simón Bolívar" por el Comité Latinoamericano de Matemática Educativa.

Entre sus obras figuran 24 artículos de investigación, 16 capítulos de libros y dos libros, sin olvidar las tesis de licenciatura y maestría que dirigió. Organizó también congresos regionales y la Escuela de Invierno en Matemática Educativa en 2017. Además, incentivó la formación, organización y proyección de grupos de investigación. Además de múltiples arbitrajes que realizó para artículos en 
revistas académicas, en particular la revista Educación Matemática le agradece su participación en todas las veces que se le invitó como árbitro.

Un espíritu tenaz, afable y reservado caracterizaba a Marcos, sus cualidades permeaban cualquier ámbito en el que tenía injerencia. Colega respetuoso, comprometido y constante, aunque en su andar llevaba "pies de plomo", como él decía. Se distinguía por su predisposición a la colaboración pues pensaba que las investigaciones tienen mayor continuidad cuando existe trabajo estructurado y conjunto: el investigador, como el ser humano, no es una isla. En este inesperado adiós, conserva el cariño de alumnos, tesistas y colegas que tuvieron la oportunidad de conocerlo, trabajar a su lado y construir conocimiento juntos. Amigo sincero, cercano e incondicional, tenía siempre palabras cálidas para esos momentos difíciles. Su ausencia deja, en todos los contextos y corazones, un lugar irremplazable.

LILIA P. AKE

Domicilio postal: Cerro de las Campanas, S/N; Colonia Las Campañas; C.P: 76010. Santiago de Querétaro, Querétaro, México

Teléfono: (442) 1921200 Ext: 71101 